\title{
Upregulation of contactin-1 expression promotes prostate cancer progression
}

\author{
BOREN WANG ${ }^{1}$, XI YANG $^{1}$, TING ZHAO ${ }^{1}$, HANGHANG DU $^{1}$, \\ TONG WANG ${ }^{1}$, SUPING ZHONG ${ }^{1}, \mathrm{BO}_{\mathrm{YANG}}{ }^{2}$ and $\mathrm{HUI} \mathrm{LI}^{3}$ \\ ${ }^{1}$ Medical School of Jishou University; ${ }^{2}$ Department of Pathology, First Affiliated Hospital of Jishou University; \\ ${ }^{3}$ Department of Microbiology and Immunology, Medical School of Jishou University, Jishou, Hunan 416000, P.R. China
}

Received March 23, 2019; Accepted November 14, 2019

DOI: $10.3892 / \mathrm{ol} .2019 .11244$

\begin{abstract}
Contactin-1 (CNTN-1) has been reported to serve an oncogenic role in several cancer types. However, detailed mechanisms describing the influence of CNTN-1 in prostate cancer progression have not yet been elucidated. The present study aimed to determine the clinical significance of CNTN-1 expression in prostate cancer progression, and also to investigate the regulatory role of CNTN-1 in the proliferation, migration and invasive ability of prostate cancer cells. The results of the present study indicated that expression levels of CNTN-1 were significantly higher in prostate cancer tissues compared with adjacent normal tissues. Moreover, a high expression level of CNTN-1 was positively correlated with tumor size, stage and metastasis, as well as a poorer prognosis in patients with prostate cancer. Furthermore, CNTN-1-knockdown in prostate cancer cells (using short hairpin RNA) resulted in the significant inhibition of cancer cell proliferation, colony formation, migration and invasiveness. Silencing of CNTN-1 expression also suppressed epithelial-mesenchymal transition in prostate cancer cells via the upregulation of E-cadherin, and the downregulation of $\mathrm{N}$-cadherin and vimentin expression. Inhibition of CNTN-1 expression also reduced the activity of the PI3K/AKT signaling pathway in prostate cancer cells. Thus, it was demonstrated that CNTN-1 expression is upregulated, and plays an oncogenic role, in prostate cancer cells. The results of the current study suggest that CNTN-1 may represent a promising therapeutic target, potentially improving the treatment of patients with prostate cancer.
\end{abstract}

\section{Introduction}

Prostate cancer is a highly prevalent malignancy and represents the second leading cause of cancer-related mortality

Correspondence to: Professor Hui Li, Department of Microbiology and Immunology, Medical School of Jishou University, 120 Renminnan Road, Jishou, Hunan 416000, P.R. China

E-mail: jishoulihui123@163.com

Key words: contactin-1, prostate cancer, proliferation, migration, invasion in elderly men $(1,2)$. Moreover, it is estimated that there would be 161,360 new prostate cancer diagnoses and 26,730 prostate cancer-associated mortalities in $2015(1,2)$. The molecular heterogeneity of prostate cancer can make its early diagnosis and treatment problematic; thus, the identification of accurate molecular biomarkers and potential therapeutic targets (at various disease stages) may result in improved patient outcome $(3,4)$. It is therefore important to investigate the molecular mechanisms underpinning the development and progression of prostate cancer, as this may catalyze the identification of novel therapeutic targets $(5,6)$.

Contactin-1 (CNTN-1), a member of the immunoglobulin superfamily is a glycosylphosphatidylinositol (GPI)-anchored neuronal membrane protein that facilitates cell adhesion $(7,8)$. It may also influence the formation of axon connections in the developing nervous system (7). Mikami et al (7) also reported that CNTN-1 was a functional receptor for neuroregulatory chondroitin sulfate-E. Additionally, Lamprianou et al (8) discovered a complex (formed from CNTN-1 and protein tyrosine phosphatase receptor type $\mathrm{Z1}$ ) that mediated the development of oligodendrocyte precursor cells. CNTN-1 is upregulated in several common types of human cancer, and promotes the progression of lung (9) and gastric cancer (10), and esophageal (11) and oral squamous cell carcinoma (12). For example, the upregulation of CNTN-1 expression is correlated with more advanced clinical stages and lymph node metastasis in patients with esophageal squamous cell carcinoma (11). Moreover, CNTN-1 expression is upregulated in oral squamous cell carcinoma, and is associated with lymph node metastasis, as well as a poor prognosis (12). Su et al (9) discovered that the knockdown of CNTN-1 expression inhibited the invasion and metastasis of lung adenocarcinoma, suggesting that it may represent a promising therapeutic target for the treatment of patients with the disease.

Furthermore, Yan et al (13) reported that the knockdown of CNTN1 inhibited stem-like, cell-mediated tumor initiation in prostate cancer. It was also reported that the overexpression of CNTN1 promoted cellular invasion in vitro, as well as enhancing xenograft tumor formation and lung metastasis in vivo (13). Taken together, these findings suggest that CNTN-1 may promote prostate cancer progression. The present study aimed to investigate the clinical significance of CNTN-1 expression in prostate cancer progression, and 
to determine the mechanism of CNTN-1 regulation of the malignant phenotypes of prostate cancer cells.

\section{Materials and methods}

Tissue collection. A total of 56 prostate cancer tissues and matched adjacent paracancerous tissues were obtained from patients with primary prostate cancer at the First Affiliated Hospital of Jishou University (Jishou, China) between April 2011 and September 2013 and stored at $-80^{\circ} \mathrm{C}$ until use. The patients were aged between 58 and 79 years (mean age, 66.5 years). The clinicopathological features of all patients are presented in Table I. Follow-up occurred for 60 months after surgery by phone calls. Written informed consent was obtained from all patients prior to surgery, and the experimental procedures were approved by the Ethics Committee of the First Affiliated Hospital of Jishou University.

Cell culture and transfection. The human prostate cell lines PC3 and LNCaP were obtained from the American Type Culture Collection. PC3 cells were cultured in 6-well plates $\left(1 \times 10^{5}\right.$ cells/well) Dulbecco's modified Eagle's medium (DMEM) and LNCaP cells were cultured in RPMI-1640 medium, both supplemented with 10\% FBS (all Thermo Fisher Scientific, Inc.); the cells were maintained at $37^{\circ} \mathrm{C}$ in a humidified atmosphere with $5 \% \mathrm{CO}_{2}$. Subsequently, both cell types were transiently transfected with either $100 \mathrm{nM}$ negative control (NC) or $100 \mathrm{nM} \mathrm{CNTN-1} \mathrm{shRNA} \mathrm{(both} \mathrm{Shanghai}$ GenePharma Co., Ltd.) using Lipofectamine ${ }^{\mathrm{TM}} 2000$ reagent (Thermo Fisher Scientific, Inc.), according to the manufacturer's protocol. The shRNA sequences were as follows: NC; 5'-UUCUCCGAACGUGUCACGUTT-3', and CNTN-1; 5'-GGUCCUUCAAUGGCUAUGUTT-3'. Subsequent experiments were conducted at $48 \mathrm{~h}$ post-transfection.

Reverse transcription-quantitative (RT-q)PCR. Total RNA was extracted from the tissues and cell lines using TRIzol ${ }^{\circledR}$ reagent (Thermo Fisher Scientific, Inc.) according to the manufacturer's protocol. The RevertAid First Strand cDNA Synthesis kit (Thermo Fisher Scientific, Inc.) was used to reverse transcribe the RNA into cDNA according to the manufacturer's protocol, for which the primer sequences are as follows: CNTN-1 forward, 5'-TGTTCAGCAAATTCATCC CA-3' and reverse, 5'-TCTACCCACTCAGGGAATGC-3'; and GAPDH forward, 5'-ACGGATTTGGTCGTATTGGGC G-3'; and reverse, 5'-CTCCTGGAAGATGGTGATGG-3'. ABI Power SYBR ${ }^{\circledR}$ Green PCR Master mix (Thermo Fisher Scientific, Inc.) was subsequently used to perform qPCR according to the manufacturer's protocol. The reaction conditions were $95^{\circ} \mathrm{C}$ for $3 \mathrm{~min}$, followed by 35 cycles of $95^{\circ} \mathrm{C}$ for $15 \mathrm{sec}, 58^{\circ} \mathrm{C}$ for $15 \mathrm{sec}$, and $72^{\circ} \mathrm{C}$ for $15 \mathrm{sec}$. The relative expression levels were quantified using the $2^{-\Delta \Delta \mathrm{Cq}}$ method (14) and normalized to those of GADPH.

Cell proliferation assay. Transfected cells $\left(5 \times 10^{4}\right.$ cells per well) were seeded into 96 -well plates, and cultured at $37^{\circ} \mathrm{C}$ for $0,24,48$ or $72 \mathrm{~h}$. Subsequently, $10 \mu \mathrm{l} \mathrm{CCK-8}$ solution (Dojindo Molecular Technologies, Inc.) was added to each well, and the cells were incubated at $37^{\circ} \mathrm{C}$ for $4 \mathrm{~h}$. The absorbance at $450 \mathrm{~nm}$ was measured using a microplate reader (Thermo Labsystems).
Colony formation assays. Transfected cells $\left(1 \times 10^{3}\right.$ cells/well $)$ were seeded into 12-well plates and cultured for 7 days. Crystal violet (0.1\%; Thermo Fisher Scientific) was then used to stain the cells before images were captured using a light microscope (magnification, $\mathrm{x} 200$ ). The number of colonies was determined using ImageJ (v. 1.46; National Institutes of health).

Wound-healing assay. Transfected cells $\left(5 \times 10^{5}\right.$ cells/well) were seeded into 6 -well plates, and cultured at $37^{\circ} \mathrm{C}$ until $\sim 100 \%$ confluence was achieved. A sterile $200-\mu 1$ pipette tip was used to scratch a wound line in each well. The transfected cells were washed twice with Dulbecco's phosphate-buffered saline (Gibco; Thermo Fisher Scientific) and resuspended in serum-free DMEM, before being incubated at $37^{\circ} \mathrm{C}$ for $24 \mathrm{~h}$. Cells were then imaged at 0 and $24 \mathrm{~h}$ using a light microscope (magnification, $\mathrm{x} 40$ ). The wound closure between the 0 to $24 \mathrm{~h}$ time points was measured using Image J (v. 1.8; NIH) and relative wound closure was determined.

Cell invasion assay. Transfected cells $\left(5 \times 10^{4}\right.$ cells/well) were resuspended in serum-free DMEM and seeded into the upper chamber of $8-\mu \mathrm{m}$ Transwell inserts (BD Biosciences), which had been pre-coated with Matrigel (BD Biosciences) at $37^{\circ} \mathrm{C}$ for $30 \mathrm{~min}$. The lower chamber was plated with DMEM supplemented with $10 \% \mathrm{FBS}$, and the cells were incubated at $37^{\circ} \mathrm{C}$ for $24 \mathrm{~h}$. The cells were then fixed using $4 \%$ paraformaldehyde at room temperature for $30 \mathrm{~min}$, and then stained using crystal violet at room temperature for $5 \mathrm{~min}$, before being imaged under a light microscope (magnification, x100). The number of invading cells was counted in five random non-overlapping fields.

Western blotting. Total protein was extracted from the transfected cells using RIPA buffer (Thermo Fisher Scientific Inc.). The total protein was quantified using a BCA method with a Pierce BCA Protein assay kit (Thermo Fisher Scientific Inc.). The proteins $(50 \mu \mathrm{g} / \mathrm{lane})$ were separated by $10 \%$ SDS-PAGE gel, and transferred onto PVDF membranes (Thermo Fisher Scientific Inc.). The membranes were blocked using 5\% non-fat milk at room temperature for $3 \mathrm{~h}$, and then incubated with rabbit anti-human antibodies against: CNTN-1 (1:500; cat. no. ab66265), E-cadherin (1:250; cat. no. ab133597), $\mathrm{N}$-cadherin (1:500; cat. no. ab76011), vimentin (1:200; cat. no. ab92547), phosphorylated (p)-PI3K (1:200; cat. no. ab182651), PI3K (1:200; cat. no. ab191606), p-AKT (1:250; cat. no. ab81283), AKT (1:500; cat. no. ab235958) and GAPDH (1:500; cat. no. ab8245) at room temperature for $3 \mathrm{~h}$, followed by further incubation with horse radish peroxidase-conjugated goat anti-rabbit secondary antibody (1:5,000; cat. no. ab6721) at room temperature for $1 \mathrm{~h}$. All antibodies were purchased from Abcam. The protein bands were visualized using the Pierce ${ }^{\mathrm{TM}}$ ECL western blotting substrate (Thermo Fisher Scientific, Inc.) and ImageJ software (v. 1.46; National Institutes of health) was used to conduct densitometric analysis.

Statistical analysis. All experiments were repeated $\geq 3$ times. The data are presented as the mean \pm SD and were analyzed using SPSS (v.20.0; IBM Corp.). Differences between 2 groups were analyzed using the paired or unpaired Student's t-test, 
Table I. Association between CNTN-1 expression and the clinicopathological characteristics of patients with prostate cancer.

\begin{tabular}{|c|c|c|c|c|}
\hline Variable & Cases $(n=56)$ & Low CNTN-1 $(n=31)$ & High CNTN-1 $(n=25)$ & P-value \\
\hline Age & & & & 0.784 \\
\hline$<65$ & 19 & 11 & 8 & \\
\hline$\geq 65$ & 37 & 20 & 17 & \\
\hline Tumor size & & & & $0.015^{\mathrm{a}}$ \\
\hline$\leq 2 \mathrm{~cm}$ & 21 & 16 & 5 & \\
\hline$>2 \mathrm{~cm}$ & 35 & 15 & 20 & \\
\hline Gleason score & & & & 0.130 \\
\hline$\leq 6$ & 17 & 12 & 5 & \\
\hline$>6$ & 39 & 19 & 20 & \\
\hline Tumor stage & & & & $0.004^{\mathrm{b}}$ \\
\hline I-II & 38 & 26 & 12 & \\
\hline III-IV & 18 & 5 & 13 & \\
\hline Lymph node metastasis & & & & $0.022^{\mathrm{a}}$ \\
\hline No & 34 & 23 & 11 & \\
\hline Yes & 22 & 8 & 14 & \\
\hline Distant metastasis & & & & $0.006^{\mathrm{b}}$ \\
\hline No & 45 & 29 & 16 & \\
\hline Yes & 11 & 2 & 9 & \\
\hline
\end{tabular}

${ }^{\mathrm{a}} \mathrm{P}<0.05,{ }^{\mathrm{b}} \mathrm{P}<0.01 . \mathrm{CNTN}-1$, contactin- 1 .

and differences between multiple groups were analyzed using one-way ANOVA followed by Tukey's post hoc test. The log-rank test was used to compare patient survival times between high- and low-CNTN1 expression groups, and the $\chi^{2}$ test was used to analyze the results presented in Table I. $\mathrm{P}<0.05$ was considered to indicate a statistically significant difference.

\section{Results}

Upregulation of CNTN-1 is associated with prostate cancer progression. In the present study, the expression levels of CNTN-1 in prostate cancer tissues were compared with those of matched adjacent paracancerous tissues. The mRNA and protein expression levels of CNTN-1 were significantly higher in prostate cancer tissues than in the corresponding adjacent tissues (Fig. $1 \mathrm{~A}$ and $\mathrm{B} ; \mathrm{P}<0.01$ ). Patients were then divided into high- and low-CNTN-1 expression groups, and the clinical significance of $\mathrm{CNTN}-1$ expression in the progression of prostate cancer was investigated. The high-expression group was significantly associated with a larger tumor size, more advanced tumor state and risk of metastasis (Table I). Moreover, it was determined that the high-CNTN-1 expression level group exhibited shorter overall survival times compared with the low-expression group, suggesting that the upregulation of CNTN-1 may predict poor prognosis in patients with prostate cancer (Fig. 1C).

Knockdown of CNTN-1 inhibits prostate cancer cell proliferation. The influence of CNTN-1 expression on prostate cancer progression was also examined in vitro. To investigate whether CNTN-1 expression was upregulated in prostate cancer cells, cells were transfected with shRNA to knockdown CNTN-1 expression. Following transfection, the mRNA and protein levels of CNTN-1 were significantly reduced when compared with those of the control group. However, transfection with NC shRNA did not affect CNTN-1 expression in prostate cancer cells (Fig. $2 \mathrm{~A}$ and $\mathrm{B} ; \mathrm{P}<0.01$ ). The function of $\mathrm{CNTN}-1$ in prostate cancer cell proliferation was then investigated. As shown in Fig. 2C-E, CNTN-1-knockdown significantly inhibited the proliferation and colony formation of prostate cancer cells $(\mathrm{P}<0.01)$, suggesting that $\mathrm{CNTN}-1$ promotes prostate cancer cell proliferation.

CNTN-1-knockdown suppresses the migration and invasion abilities of prostate cancer cells. To further characterize the function of CNTN-1 in prostate cancer metastasis, wound-healing and Transwell assays were performed to examine the effects of CNTN-1-knockdown on the migration and invasiveness of prostate cancer cells. As shown in Fig. 3A and B, prostate cancer cell migration was significantly inhibited in the CNTN-1-knockdown group, compared with the control group $(\mathrm{P}<0.01)$. Knockdown of $\mathrm{CNTN}-1$ significantly suppressed the invasive capacity of PC3 and LNCaP cells (Fig. 3C and D; $\mathrm{P}<0.01$ ). Therefore, CNTN-1 is suggested to promote the regulation of migration and invasion in prostate cancer cells.

Inhibition of CNTN-1 represses epithelial-mesenchymal transition (EMT) and PI3K/AKT signaling in prostate cancer cells. The effect of CNTN-1-knockdown on EMT (a mechanism facilitating cancer cell migration and invasion) in prostate cancer cells was also investigated. As exhibited in 


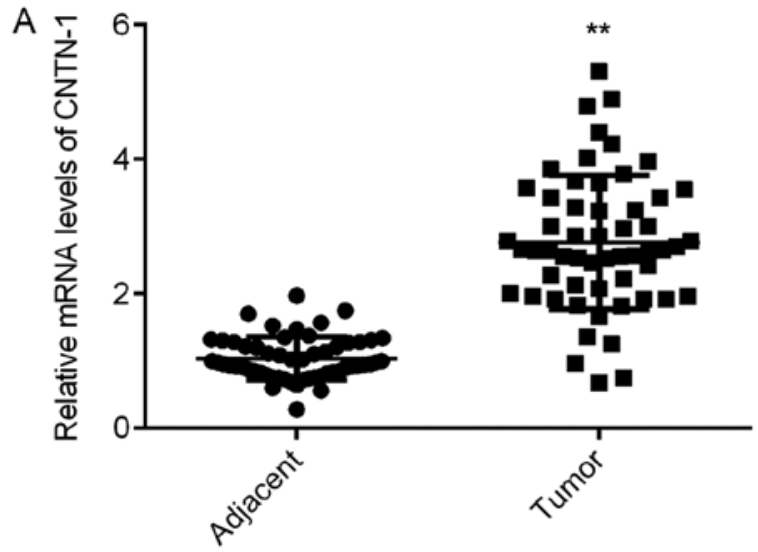

B
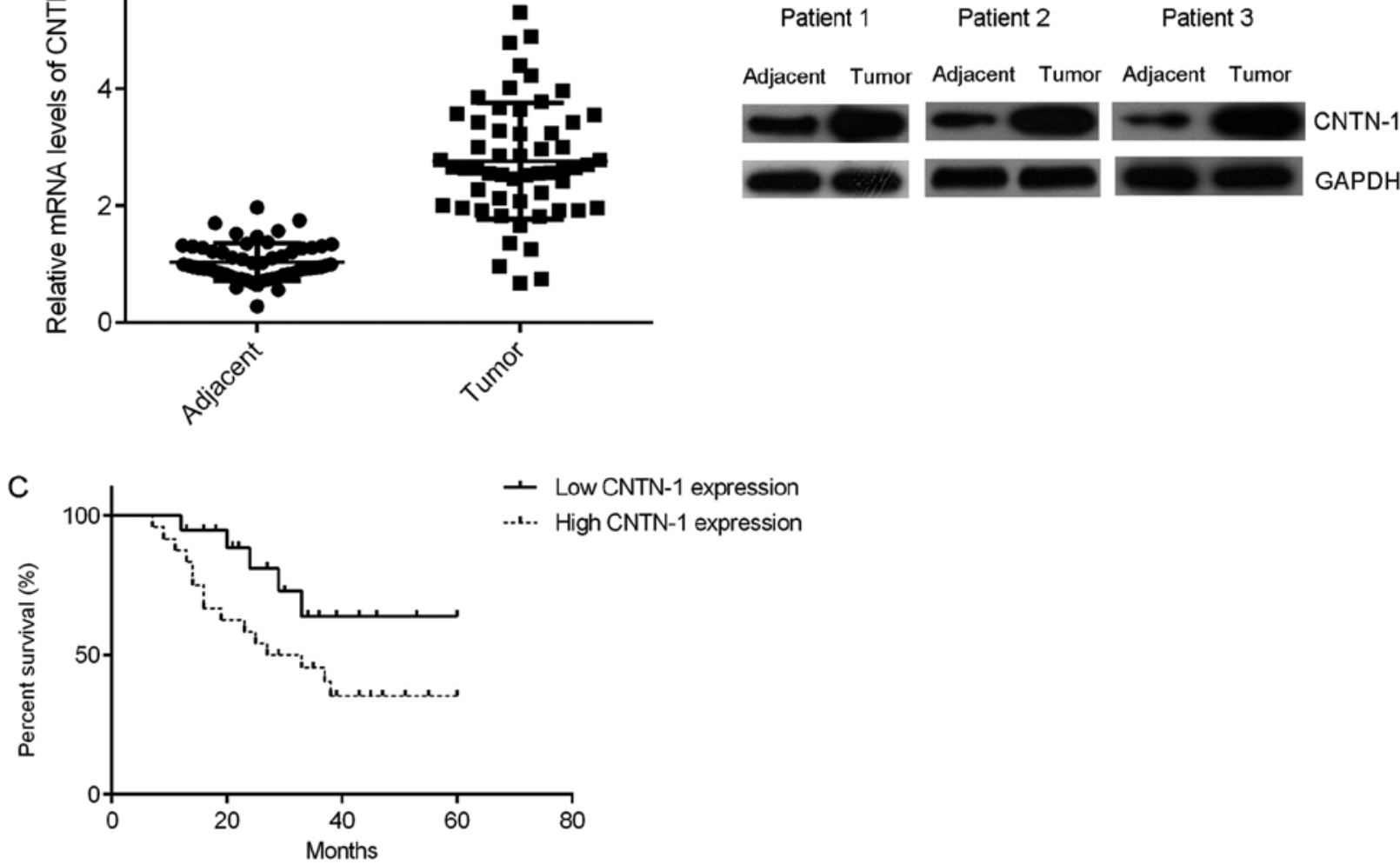

Figure 1. Upregulation of CNTN-1 is associated with prostate cancer progression. Results of (A) reverse transcription-quantitative PCR and (B) western blotting indicated that the CNTN-1 expression was increased at the mRNA and protein level in prostate cancer tissues, compared with adjacent paracancerous tissues. ${ }^{* *} \mathrm{P}<0.01$ vs. adjacent paracancerous tissues. (C) Patients with prostate cancer with high CNTN-1 expression levels exhibited shorter overall survival times than those with low expression levels. CNTN-1, contactin-1.

Fig. 4A and B, knockdown of CNTN-1 significantly increased the expression of E-cadherin, but decreased the protein levels of $\mathrm{N}$-cadherin and Vimentin in prostate cancer cells $(\mathrm{P}<0.01)$, indicating that EMT was inhibited. Therefore, it was suggested that CNTN-1-knockdown may suppress prostate cancer metastasis via the inhibition of EMT.

The molecular mechanism of CNTN-1 in prostate cancer progression was then investigated. PI3K/AKT signaling has been reported to influence prostate tumor growth and metastasis. Therefore, the function of CNTN-1 in the regulation of the PI3K/AKT signaling pathway was assessed. As indicated in Fig. 4C and D, the expression levels of phosphorylated $\mathrm{PI} 3 \mathrm{~K}$ and AKT were significantly reduced in the shCNTN-1 group compared with those the shNC group, indicating that CNTN-1-knockdown reduced PI3K/AKT signaling in prostate cancer cells $(\mathrm{P}<0.01)$. Therefore, the results of the current study suggest that the PI3K/AKT signaling pathway may be involved in the function of CNTN-1, by regulating the malignant phenotypes of prostate cancer cells.

\section{Discussion}

The function and clinical significance of CNTN-1 expression in prostate cancer has not yet been fully elucidated. In the present study, it was observed that the expression level of CNTN-1 was significantly higher in prostate cancer tissues compared with adjacent paracancerous tissues. Moreover, high expression of CNTN-1 was positively correlated with cancer progression, as well as poor prognosis in patients with prostate cancer. CNTN-1-knockdown resulted in significant inhibitory effects on prostate cancer cell proliferation, colony formation, migration and invasiveness. Moreover, the CNTN-1-knockdown inhibited EMT and modulated $\mathrm{PI} 3 \mathrm{~K} / \mathrm{AKT}$ signaling in prostate cancer cells.

CNTN-1 has been discovered to promote prostate cancer cell invasion in vitro, as well as tumor growth and lung metastasis in vivo (13). In the present study, the expression pattern and function of CNTN-1 in prostate cancer was investigated, and the data suggested that the expression levels of CNTN-1 were significantly higher in prostate cancer tissues compared with those in adjacent paracancerous tissues. Moreover, upregulation of CNTN-1 expression was significantly associated with a larger tumor size, a more advanced clinical stage and metastasis in patients with prostate cancer. Consistent with the results of the present study, Yan et al (13) reported that the expression level of CNTN-1 was significantly higher in prostate cancer cells from primary tumors, lymph nodes and bone metastases, compared with paracancerous prostate gland tissues. The present study indicated that patients with high-CNTN-1 expression exhibited shorter overall survival times when compared with the low-expression group, suggesting that CNTN-1 expression may be used as a predictive biomarker of prostate cancer. Yan et al also showed that following radical prostatectomy, CNTN1 expression was associated with a shorter recurrence-free survival time in patients with prostate cancer (13). In the current study, transfection with shRNA 
A
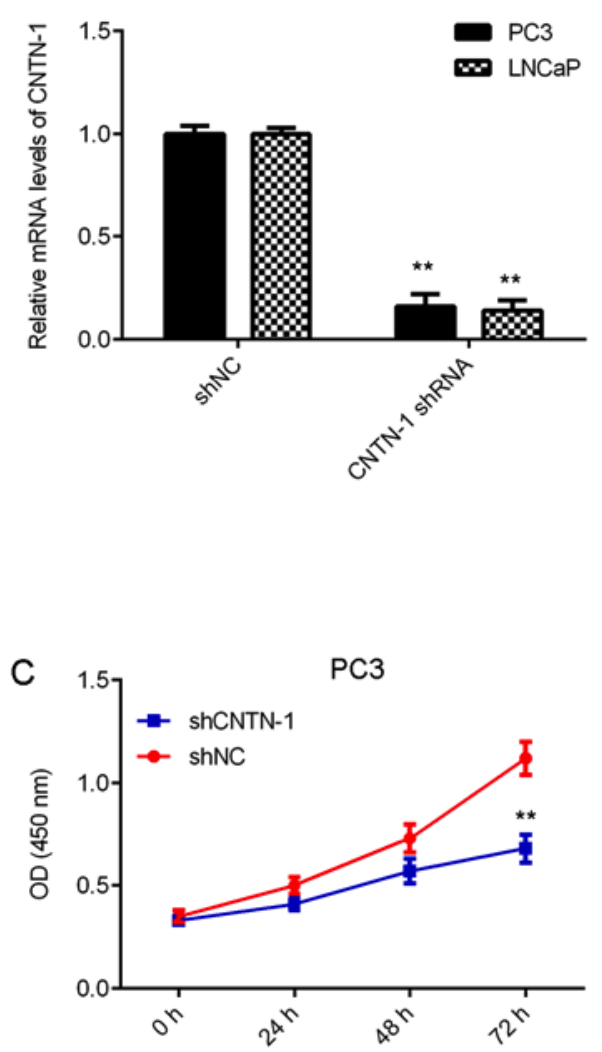

E

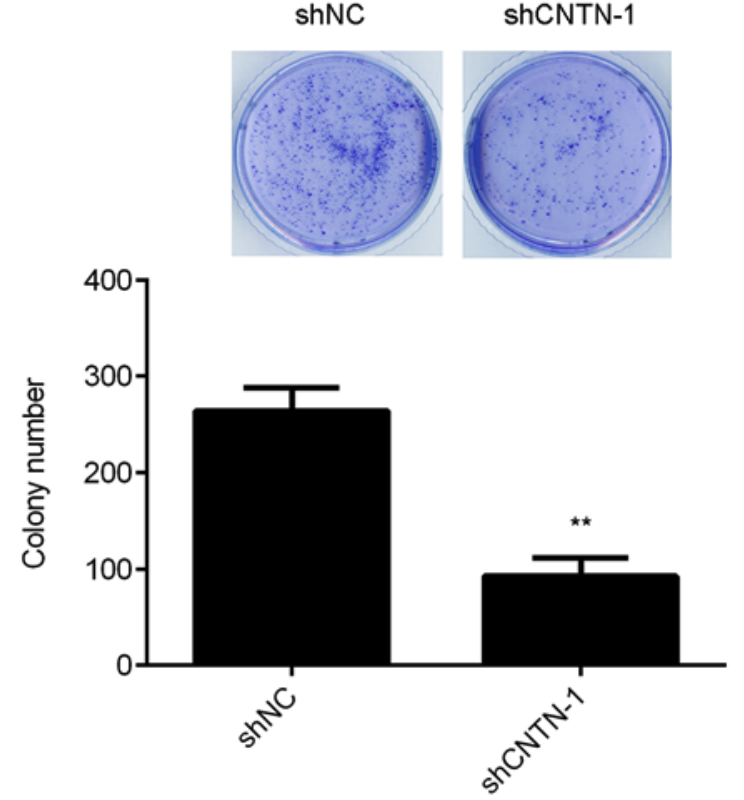

B

PC3

LNCaP

shNC ShCNTN-1 ShNC ShCNTN-1

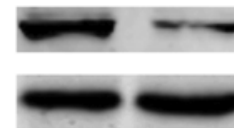

$=\mathrm{CNTN}-1$

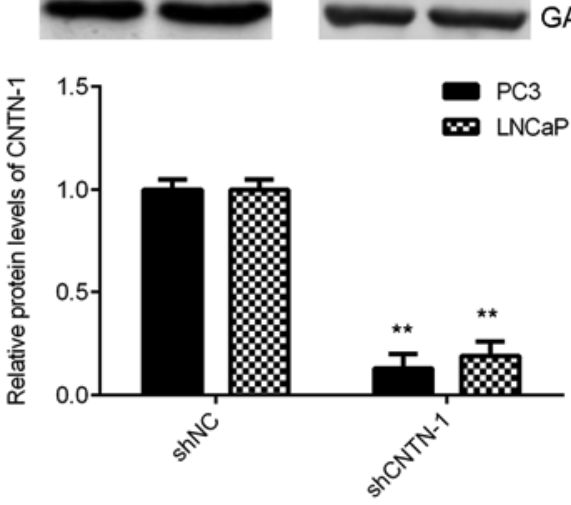

D

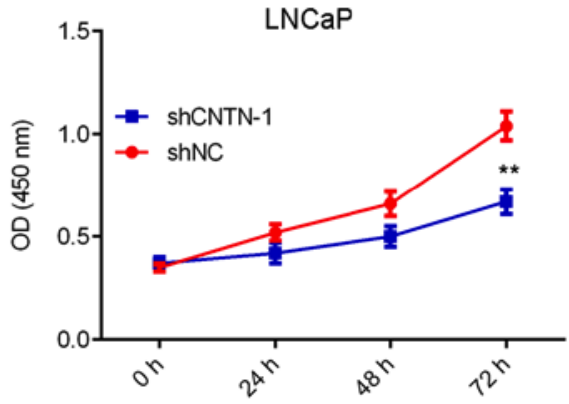

F

LNCaP

shNC

shCNTN-1
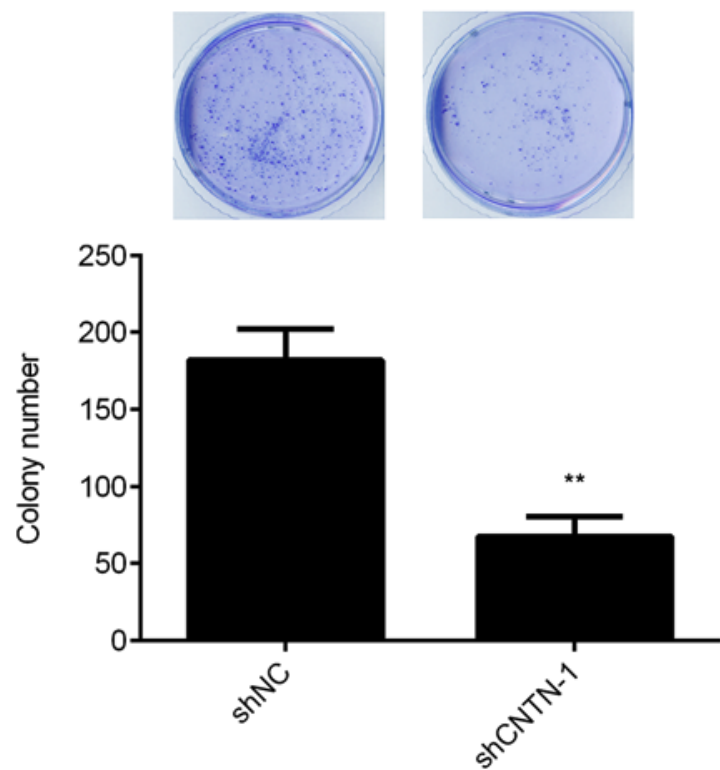

Figure 2. CNTN-1-knockdown inhibits prostate cancer cell proliferation. PC3 and LNCaP prostate cancer cell were transfected with negative control shRNA or CNTN-1 shRNA. Following transfection, (A) reverse transcription-quantitative PCR and (B) western blotting were used to examine the mRNA and protein expression of CNTN-1. A CCK-8 assay was performed to study the proliferation of (C) PC3 and (D) LNCaP cells. The colony formation rate of (E) PC3 and (F) LNCaP was also examined. ${ }^{* *} \mathrm{P}<0.01$ vs. shNC. CNTN-1, contactin-1; shRNA, short hairpin RNA; shNC, short hairpin negative control; OD, optimal density.

was used to knockdown the expression of CNTN-1 in prostate cancer cell lines, which resulted in significantly reduced proliferation, colony formation, migration and invasiveness.
This further supports the hypothesis that CNTN-1 promotes the progression of prostate cancer, and suggests that CNTN-1 may represent a promising therapeutic target for the treatment 
A

PC3

$\mathrm{Oh}$
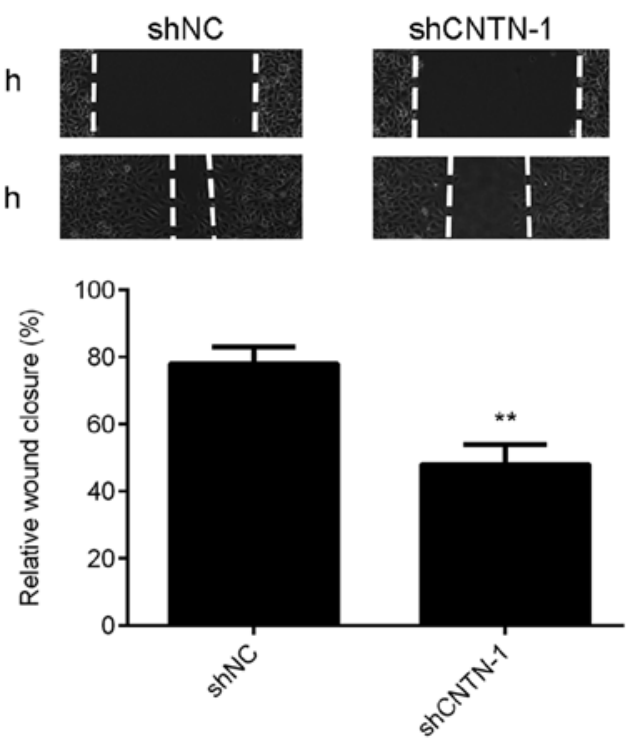

C

PC3

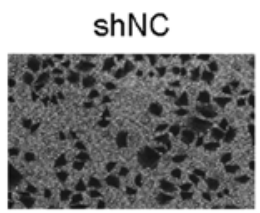

shCNTN-1
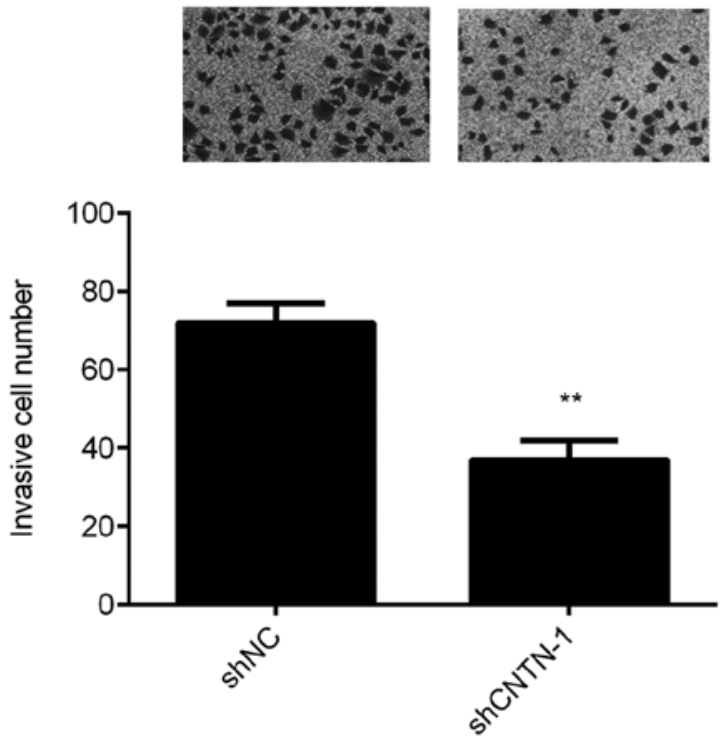

B
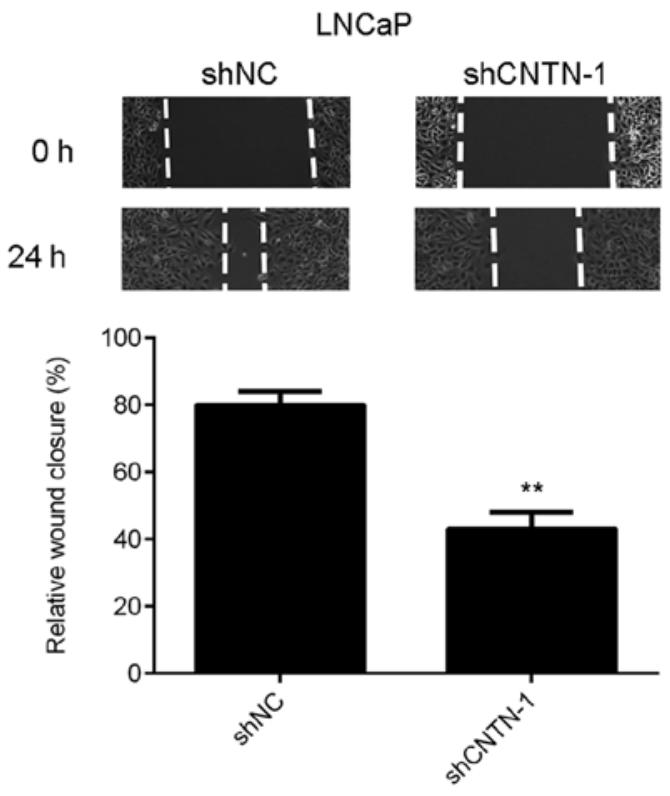

D

$\operatorname{shNC}$

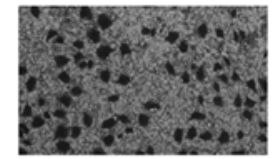

shCNTN-1
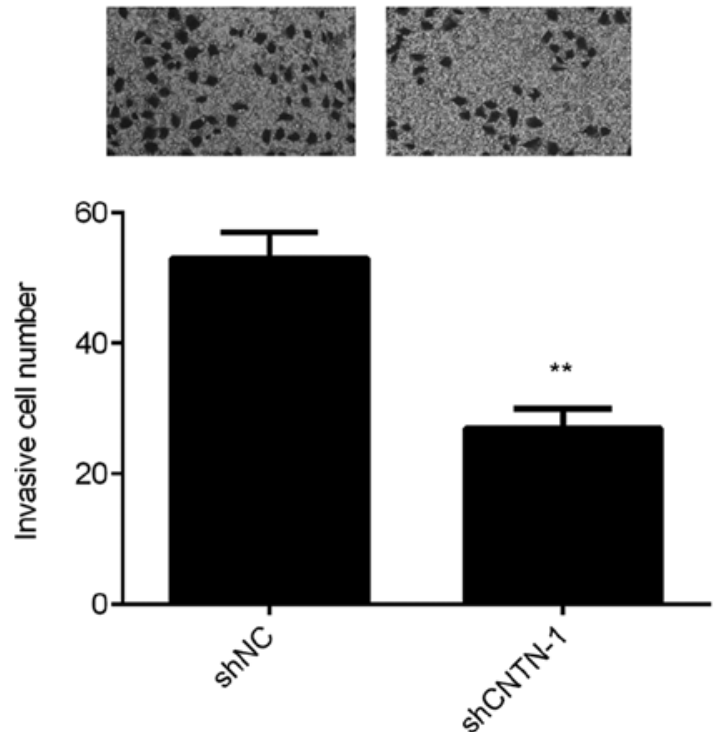

Figure 3. CNTN-1-knockdown suppresses the migration and invasiveness of prostate cancer cells. PC3 and LNCaP prostate cancer cells were transfected with negative control shRNA or CNTN-1 shRNA. Following transfection, a wound-healing assay was performed on (A) PC3 and (B) LNCaP cells. An invasion assay was also conducted on (C) PC3 and (D) LNCaP cells. ${ }^{* *} \mathrm{P}<0.01$ vs. shNC. CNTN-1, contactin-1; shRNA, short hairpin RNA; shNC, short hairpin negative control; shCNTN-1, short hairpin RNA against contactin-1; OD, optimal density.

of the disease. Yan et al (13) used the DU145 cell line to study the function of CNTN-1 in vitro. In the present study, two different cell lines (PC3 and LNCaP) were used, providing further validation of these results (13). Moreover, in the study conducted by Yan et al (13), only CNTN-1 cell invasion was investigated. In the present study wound-healing assays were performed to further elucidate the function of CNTN-1 in prostate cancer cell migration.

EMT is tightly regulated by several internal and external stimuli that orchestrate the transition from an epithelial-like to a mesenchymal phenotype (15-17). EMT facilitates tumor cell invasiveness and metastatic capacity, and is thus a principal mediator of cancer progression and metastasis (18-20).
Yan et al (13) only detected the expression of E-cadherin, and thus did not reveal the function of CNTN-1 in EMT in prostate cancer. In the present study, elucidation of the role of CNTN-1 in EMT was a key objective. Thus, the effect of CNTN-1-knockdown significantly increased the expression of E-cadherin, while inhibiting the expression of $\mathrm{N}$-cadherin and vimentin in prostate cancer cells, indicating that EMT was suppressed. The current findings indicated that the CNTN-1-mediated promotion of prostate cancer cell migration and invasion might be the result of EMT regulation.

The PI3K/AKT signaling pathway promotes tumor cell proliferation, migration and invasion, and EMT in multiple human cancers (21-24). In the present study, it was 
A
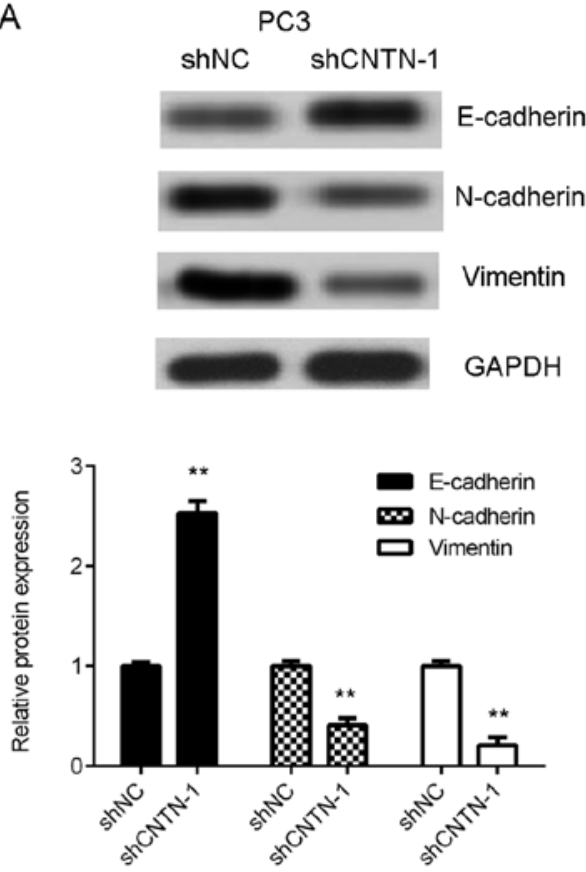

C

PC3

shNC shCNTN-1
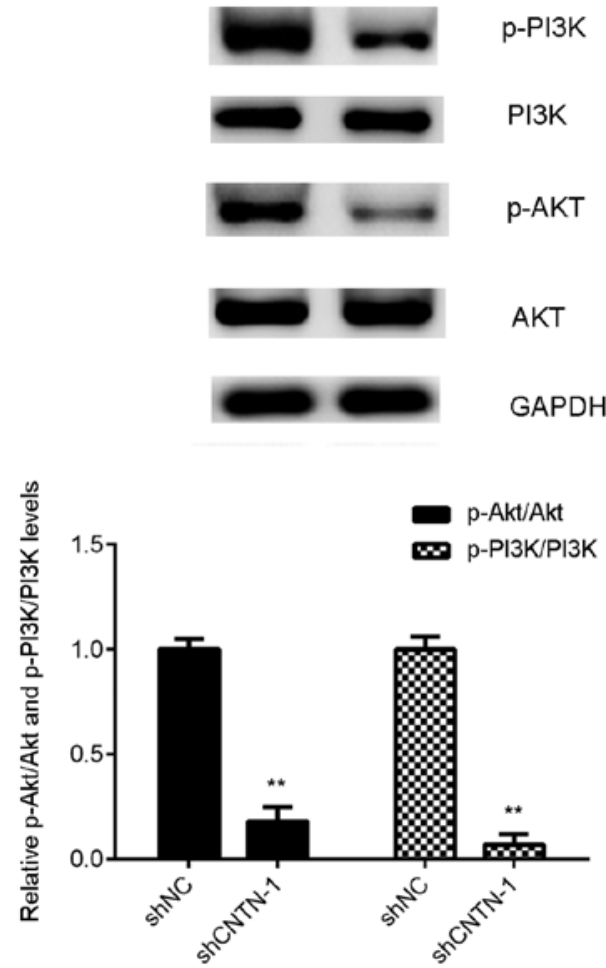

B
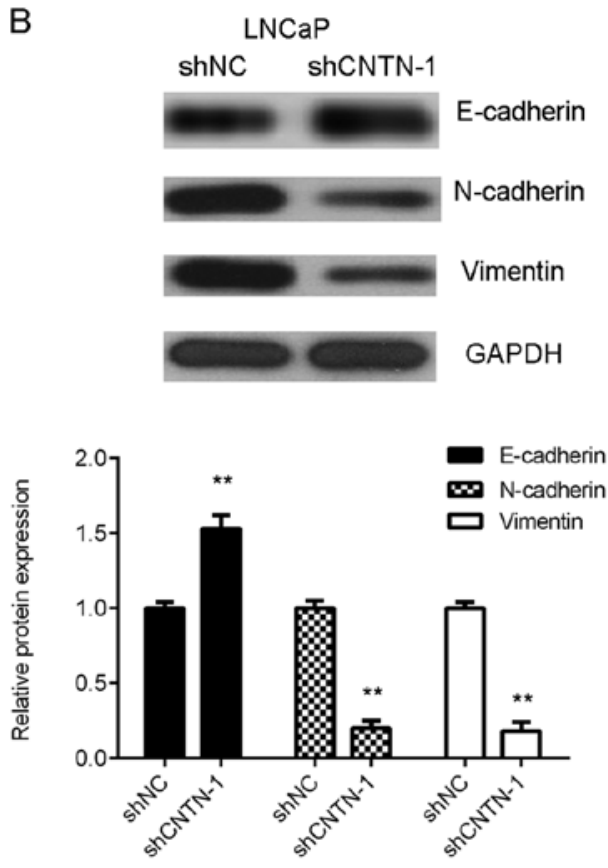

D
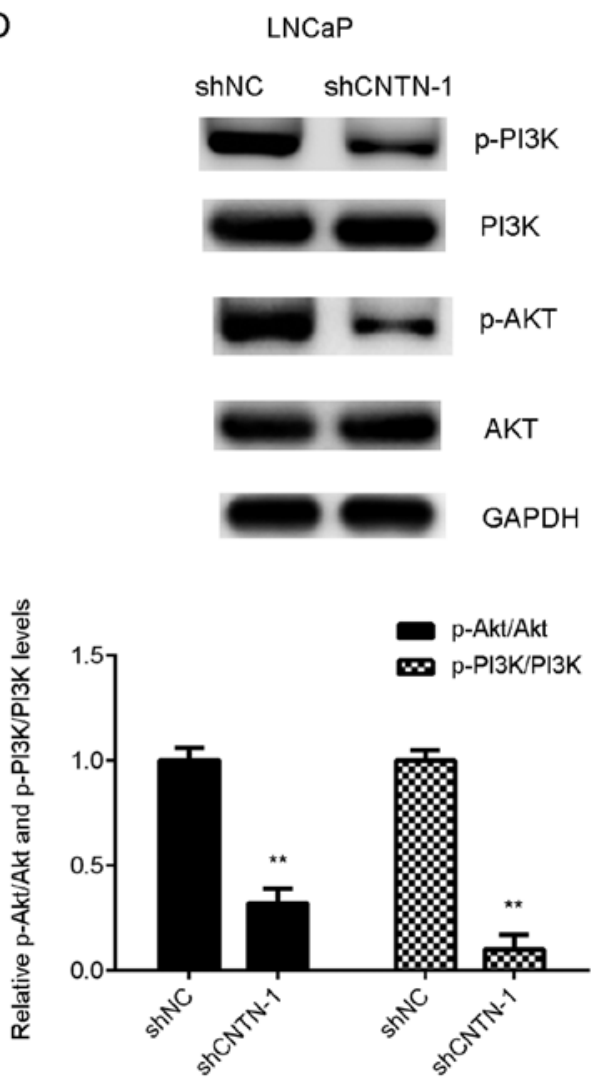

Figure 4. Inhibition of CNTN-1 represses EMT and the activity of the PI3K/AKT signaling in prostate cancer cells. Western blotting was performed to determine the expression levels of E-cadherin, N-cadherin and vimentin in order to investigate the effect of CNTN-1-knockdown on EMT in (A) PC3 and (B) LNCaP cells. Expression levels of PI3K and AKT were then determined to investigate the effect of CNTN-1-knockdown on the activity of the PI3K/AKT pathway in (C) PC3 and (D) LNCaP cells. ${ }^{* *} \mathrm{P}<0.01$ vs. shNC. CNTN-1, contactin-1; shRNA, short hairpin RNA; shNC, short hairpin negative control; shCNTN-1, short hairpin RNA against contactin-1; EMT, epithelial-mesenchymal transition.

discovered that CNTN-1-knockdown significantly inhibited the PI3K/AKT signaling pathway in prostate cancer cells. Similar findings have also been reported in lung cancer. For instance, Zhang et al (25) reported that CNTN-1- enhanced chemoresistance in lung adenocarcinoma via the promotion of EMT, by activating the PI3K/AKT signaling pathway. Moreover, Yan et al reported that CNTN-1 inhibited E-cadherin expression via the activation of AKT in lung 
cancer (26). Therefore, the interaction between CNTN-1 and the PI3K/AKT signaling pathway may represent a commonality shared by multiple cancer types. Animal experiments may help to further clarify the exact function, and validate the regulatory mechanisms of CNTN-1 in prostate cancer, in vivo.

In summary, the present study demonstrated that CNTN-1 was significantly upregulated in prostate cancer compared with adjacent paracancerous tissues, and that the knockdown of CNTN-1 inhibited proliferation, migration, invasiveness and EMT in prostate cancer cells. Taken together, the results suggest that CNTN-1 may represent a potential therapeutic target for the treatment of prostate cancer.

\section{Acknowledgements}

Not applicable.

\section{Funding}

Not applicable.

\section{Availability of data and materials}

All data generated or analyzed during the present study are included in this published article.

\section{Authors' contributions}

HL and BW designed the study and wrote the manuscript. BW, XY, TZ, HD, TW and SZ performed all of the experiments. BY conducted the statistical analysis. All authors read and approved the final manuscript.

\section{Ethics approval and consent to participate}

The present study was approved by the Ethics Committee of First Affiliated Hospital of Jishou University, Jishou, China. Written informed consent was obtained from each participant, prior to surgery.

\section{Patient consent for publication}

Not applicable.

\section{Competing interests}

The authors declare that they have no competing interests.

\section{References}

1. Siegel RL, Miller KD and Jemal A: Cancer statistics, 2017. CA Cancer J Clin 67: 7-30, 2017.

2. Siegel RL, Miller KD and Jemal A: Cancer statistics, 2015. CA Cancer J Clin 65: 5-29, 2015

3. Misawa A and Inoue S: Estrogen-related receptors in breast cancer and prostate cancer. Front Endocrinol (Lausanne) 6: 83, 2015.

4. Mao Y, Li K, Lu L, Si-Tu J, Lu M and Gao X: Overexpression of Cdc20 in clinically localized prostate cancer: Relation to high Gleason score and biochemical recurrence after laparoscopic radical prostatectomy. Cancer Biomark 16: 351-358, 2016.
5. Xiong W, Huang C, Deng H, Jian C, Zen C, Ye K, Zhong Z, Zhao X and Zhu L: Oncogenic non-coding RNA NEAT1 promotes the prostate cancer cell growth through the SRC3/IGF1R/AKT pathway. Int J Biochem Cell Biol 94: 125-132, 2018.

6. Guo W, Keener AL, Jing Y, Cai L, Ai J, Zhang J, Fisher AL, Fu G and Wang Z: FOXA1 modulates EAF2 regulation of AR transcriptional activity, cell proliferation, and migration in prostate cancer cells. Prostate 75: 976-987, 2015.

7. Mikami T, Yasunaga D and Kitagawa H: Contactin-1 is a functional receptor for neuroregulatory chondroitin sulfate-E. J Biol Chem 284: 4494-4499, 2009.

8. Lamprianou S, Chatzopoulou E, Thomas JL, Bouyain S and Harroch S: A complex between contactin-1 and the protein tyrosine phosphatase PTPRZ controls the development of oligodendrocyte precursor cells. Proc Natl Acad Sci USA 108: 17498-17503, 2011.

9. Su JL, Yang CY, Shih JY, Wei LH, Hsieh CY, Jeng YM, Wang MY, Yang PC AND Kuo ML: Knockdown of contactin-1 expression suppresses invasion and metastasis of lung adenocarcinoma. Cancer Res 66: 2553-2561, 2006.

10. Yu JW, Wu SH, Lu RQ, Wu JG, Ni XC, Zhou GC, Jiang HG, Zheng LH, Li XQ, Du GY and Jiang BJ: Expression and significances of contactin-1 in human gastric cancer. Gastroenterol Res Pract 2013: 210205, 2013.

11. Liu P, Chen S, Wu W, Liu B, Shen W, Wang F, He X and Zhang S: Contactin-1 (CNTN-1) overexpression is correlated with advanced clinical stage and lymph node metastasis in oesophageal squamous cell carcinomas. Jpn J Clin Oncol 42: 612-618, 2012.

12. Wu HM, Cao W, Ye D, Ren GX, Wu YN and Guo W: Contactin 1 (CNTN1) expression associates with regional lymph node metastasis and is a novel predictor of prognosis in patients with oral squamous cell carcinoma. Mol Med Rep 6: 265-270, 2012.

13. Yan J, Ojo D, Kapoor A, Lin X, Pinthus JH, Aziz T, Bismar TA, Wei F, Wong N, De Melo J, et al: Neural cell adhesion protein CNTN1 promotes the metastatic progression of prostate cancer. Cancer Res 76: 1603-1614, 2016.

14. Livak KJ and Schmittgen TD: Analysis of relative gene expression data using real-time quantitative PCR and the 2(-Delta Delta C(T)) method. Methods 25: 402-408, 2001.

15. Singh M, Yelle N, Venugopal C and Singh SK: EMT: Mechanisms and therapeutic implications. Pharmacol Ther 182: 80-94, 2018.

16. Smith BN and Bhowmick NA: Role of EMT in metastasis and therapy resistance. J Clin Med 5: pii: E17, 2016.

17. Beuran M, Negoi I, Paun S, Ion AD, Bleotu C, Negoi RI and Hostiuc S: The epithelial to mesenchymal transition in pancreatic cancer: A systematic review. Pancreatology 15: 217-225, 2015.

18. Wu Y, Sarkissyan M and Vadgama JV: Epithelial-mesenchymal transition and breast cancer. J Clin Med 5: pii: E13, 2016.

19. Jiang Z, Song Q, Zeng R, Li J, Li J, Lin X, Chen X, Zhang J and Zheng Y: MicroRNA-218 inhibits EMT, migration and invasion by targeting SFMBT1 and DCUN1D1 in cervical cancer. Oncotarget 7: 45622-45636, 2016.

20. Barrette K, Van Kelst S, Wouters J, Marasigan V, Fieuws S, Agostinis P, van den Oord J and Garmyn M: Epithelial-mesenchymal transition during invasion of cutaneous squamous cell carcinoma is paralleled by AKT activation. $\mathrm{Br} \mathrm{J}$ Dermatol 171: 1014-1021, 2014.

21. Sathe A and Nawroth R: Targeting the PI3K/AKT/mTOR pathway in bladder cancer. Methods Mol Biol 1655: 335-350, 2018.

22. Murthy D, Attri KS and Singh PK: Phosphoinositide 3-kinase signaling pathway in pancreatic ductal adenocarcinoma progression, pathogenesis, and therapeutics. Front Physiol 9: 335, 2018.

23. Zhao HF, Wang J, Shao W, Wu CP, Chen ZP, To ST and Li WP: Recent advances in the use of PI3K inhibitors for glioblastoma multiforme: Current preclinical and clinical development. Mol Cancer 16: 100, 2017.

24. Zhang J, Yu XH, Yan YG, Wang $\mathrm{C}$ and Wang WJ: PI3K/Akt signaling in osteosarcoma. Clin Chim Acta 444: 182-192, 2015.

25. Zhang R, Sun S, Ji F, Liu C, Lin H, Xie L, Yang H, Tang W, Zhou Y, Xu J and Li P: CNTN-1 enhances chemoresistance in human lung adenocarcinoma through induction of epithelial-mesenchymal transition by targeting the PI3K/Akt pathway. Cell Physiol Biochem 43: 465-480, 2017.

26. Yan J, Wong N, Hung C, Chen WX and Tang D: Contactin-1 reduces E-cadherin expression via activating AKT in lung cancer. PLoS One 8: e65463, 2013.

This work is licensed under a Creative Commons Attribution-NonCommercial-NoDerivatives 4.0 International (CC BY-NC-ND 4.0) License. 